\title{
ESTUDIO EXPLORATORIO DE LA CALIDAD EN EL PERIODISMO DIGITAL EN CASTILLA-LA MANCHA
}

\section{EXPLORATORY RESEARCH OF QUALITY IN DIGITAL JOURNALISM IN CASTILLA-LA MANCHA}

\author{
Belén Galletero Campos \\ Vanesa Saiz Echezarreta \\ Universidad de Castilla-La Mancha. Cuenca/España \\ Belen.galletero@uclm.es \\ Vanesa.saiz@uclm.es
}

Recibido/Received: 05/06/2018

Modificado/Modified: 15/07/2018

Aceptado/Accepted: 22/09/2018

\section{RESUMEN}

Esta investigación es una aproximación a los estándares de calidad en el periodismo digital de CastillaLa Mancha. Su importancia radica en que esta comunidad autónoma es escenario de una acelerada migración desde los medios de comunicación tradicionales a los medios creados en internet. Considerando que los cibermedios ocupan ahora una posición privilegiada, es pertinente evaluar si mantienen las buenas prácticas exigibles al periodismo como actividad social de servicio público. Con este objetivo, se aplican unos parámetros ad hoc, diseñados a partir de la revisión bibliográfica sobre calidad en medios digitales. La metodología se ha aplicado a una muestra de 20 medios digitales que se someten al control de OJD y han recibido ingresos de la administración regional a través de publicidad institucional. El diagnóstico ha puesto de relieve prácticas que alertan sobre una devaluación en el ejercicio periodístico y, por ende, en la calidad de la información que transmiten estos medios.

\section{PALABRAS CLAVE}

Periodismo; innovación; mala praxis; Castilla-La Mancha; servicio público.

\section{SUMARIO}

1. Introducción. 2. El concepto de calidad en los medios de comunicación. 3. Metodología. 4. Resultados. 5. Conclusiones. Bibliografía.

\begin{abstract}
This research is an approximation to the quality standards in digital journalism in Castilla-La Mancha. Its importance lies in the fact that this region is the scene of an accelerated migration from traditional to new on line media. Considering that cybermedia are now in a privileged position, it is convenient to evaluate whether they maintain the good practices required for journalism as a social and public service activity. With this objective, ad hoc parameters are applied, designed from the bibliographic review on quality in digital media. This method has been applied to a sample of 20 digital media that are examined by OJD control and have received revenues from the regional administration through institutional
\end{abstract}


advertising. The diagnosis has highlighted some questionable practices that warn of a devaluation in the journalistic exercise and, therefore, in the quality of the information transmitted by these media.

\section{KEYWORDS}

Journalism; Innovation; Castilla-La Mancha; Bad Praxis; Public Service.

\section{CONTENTS}

1. Introduction. 2. The concept of quality in the media. 3. Methodology. 4. Results. 5. Conclusions. References.

\section{INTRODUCCIÓN}

El Observatorio de la Comunicación de Castilla-La Mancha - MediaCom (Facultad de Periodismo-UCLM) nació en 2015 con el objetivo de realizar rastreos sistemáticos de los medios de proximidad en la citada región, de tal manera que se pudiera evaluar su evolución reciente. Su puesta en marcha constituye el primer intento de iniciar una línea de investigación con continuidad acerca de los medios de comunicación en esta comunidad autónoma, ya que las contribuciones académicas dedicadas a su estudio son escasas. Sería pertinente reflexionar en qué medida la falta de interés académico y de estudios exploratorios guarda relación con la ecología de los medios regionales, caracterizada por la precariedad y la alta dependencia de la financiación pública (Galletero, 2018). Si bien el historiador Isidro Sánchez ha dedicado monografías a la historia y la estructura de la prensa a finales del siglo XIX y principios del XX (Sánchez, 1991), y a la prensa en Cuenca (López-Villaverde y Sánchez, 1998) y Ciudad Real (Ortega y Sánchez, 2007), no se han producido con posterioridad trabajos sobre el conjunto de los medios, con la excepción del análisis estructural realizado por Javier Díaz Revorio en 2002. Sí se han editado algunos estudios puntuales fuera del ámbito científico y centrados en el perfil de los profesionales, como el realizado por el Consejo Regional de Relaciones Laborales bajo el título La situación laboral de los profesionales de los medios de comunicación en Castilla-La Mancha (2011) y otro sobre la situación de las mujeres periodistas en Castilla-La Mancha, financiado por el Instituto de la Mujer (2004). Este vacío presenta una oportunidad para indagar en la manera en que los medios locales y regionales se han ido adaptando a los trascendentales cambios que han sufrido el mercado de la comunicación y los hábitos de consumo en las últimas décadas. Su interés se asienta en que los medios de comunicación están directamente relacionados con la acción política y el ejercicio democrático: en cierta medida, operan como un indicador de la implicación de la ciudadanía y la calidad del debate público, una relación sobre la que se mantiene viva la reflexión expuesta por, entre otros, John Dewey en 1927 (2004).

\subsection{Justificación}

Ante la ausencia de directorios oficiales y de estudios recientes, el primer trabajo del Observatorio en 2015 fue realizar un censo de los medios en activo para su posterior catalogación en una base de datos, identificando cuestiones como su localización, año de inicio, tipo de propiedad y datos de contacto, así como aspectos específicos relativos a cada soporte (publicaciones impresas, radio, televisión y medios digitales). Una de las conclusiones obtenidas en la primera aproximación, cuyos resultados dieron lugar al trabajo Los medios de comunicación en Castilla-La Mancha. Informe 2015 (Laguna et al., 2016), 
fue la acelerada migración al entorno digital que se ha producido en este ecosistema mediático en los últimos diez años. De 29 medios digitales documentados en el año 2007 (Morate, 2010) hemos pasado a 86 que funcionan en la actualidad, de los cuales 63 son nativos digitales, es decir, no tienen un modelo matricial en papel. En contraste, y a raíz de la crisis económica y estructural de la prensa (De Bustos y Casado, 2010; De Mateo, Bergés y Garnatxe, 2010; Díaz Nosty, 2013), desde 2008 han desaparecido algunas de las publicaciones en papel más longevas de la región (Galletero, 2018), como La Verdad de Albacete (1973-2013), El Día de Cuenca (1984-2013) o El Decano (antiguo Flores y Abejas con origen en 1894), mientras otras se han transformado, como el diario ciudadrealeño Lanza, que en 2017 ha pasado a ser un diario digital complementado con un semanario impreso gratuito. Tanto es así, que a día de hoy sólo se editan tres cabeceras diarias en papel, lo que convierte al periodismo impreso de la región en una práctica en extinción. Las consecuencias de este fenómeno están aún sin estudiar: se desconocen de forma pormenorizada los hábitos informativos de aquella parte de la población que no consume de manera asidua medios digitales y los efectos que la brecha digital genera en las formas de participación en el espacio público y en la acción política.

Lo cierto es que un nuevo contexto se ha consolidado. El ritmo de crecimiento de medios en internet, intensificado entre 2013 y 2015 - coincidiendo con los cierres de las grandes empresas periodísticas, no se ha detenido. De igual modo, si nos fijamos en la evolución de la penetración que han registrado los medios en la comunidad autónoma en los últimos diez años, se confirma el descenso de la audiencia de la prensa, muy por debajo de los demás medios, mientras el uso de internet supera a todos los demás, con un crecimiento acusado a partir de 2009 y una progresión más pronunciada que en la radio y la televisión, cuyo consumo permanece estable.

Ilustración 1: Evolución de la penetración de los medios de comunicación en Castilla-La Mancha

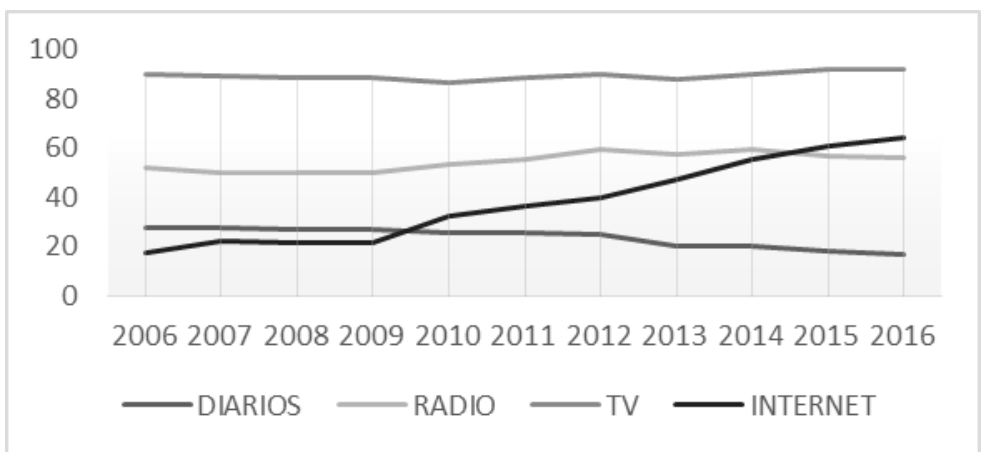

Fuente: Marco General de los Medios en España 2007-2017. Ediciones anuales

El escenario actual, por tanto, plantea nuevos retos. Las bajas barreras de entrada del mercado digital se han traducido en una oportunidad para periodistas (y no periodistas) de lanzar sus propios medios, con un modelo empresarial alejado de las plantillas y las empresas editoras que marcaron la época de las rotativas. En su lugar, se han implantado modelos de redacciones exiguas, compuestas a menudo por trabajadores y trabajadoras autónomas, a veces con sólo uno o dos periodistas para elaborar la información. Existen normativas de ámbito estatal y autonómico para la regulación y explotación de radios y televisiones pero no 
así para la prensa (Díaz Revorio, 2002), menos aún para los medios en internet, donde, por naturaleza, todo es más desordenado. De ahí la conveniencia de abordar la reflexión teórica sobre nuevas praxis y pautas deontológicas adaptadas al nuevo entorno digital (Cruz y Suárez Villegas, 2017).

\subsection{Objetivos}

El objetivo principal de esta contribución es determinar cómo es el prototipo de cibermedio que opera en este territorio y valorar algunos indicadores acerca de su calidad periodística en aras de establecer las condiciones mínimas que debe cumplir un producto para ser categorizado como un medio de comunicación informativo.

Los objetivos específicos son:

1) Construir indicadores desde la observación para fomentar la calidad en los medios digitales de esta comunidad.

2) Comprobar qué indicadores obtienen mayor grado de cumplimiento y cuáles presentan margen de mejora.

Esta línea de trabajo se proyecta a largo plazo, con seguimientos anuales, adoptando las metodologías propias de los observatorios de comunicación (Rosique y Barranquero, 2016). Por todo ello, lo que se presenta a continuación es una propuesta para fijar el método y los resultados de una primera exploración de tipo descriptivo que facilite seguimientos posteriores. Se busca, en última instancia, participar en el debate sobre qué constituye hoy en día un medio de comunicación local y cuáles serían los estándares mínimos exigibles para percibir financiación pública, así como favorecer la implantación de sistemas de rendición de cuentas que contribuyan a garantizar el derecho constitucional a la información y promuevan la función de servicio público de los medios informativos. En este aspecto, estudios posteriores pueden determinar con mayor nivel de precisión a partir de la evolución en los datos si el dinero público se traduce finalmente en una mejora de los indicadores. Así, este tipo de investigaciones están llamadas a generar una cultura de colaboración entre universidad, administraciones y medios para fomentar la credibilidad, la confianza y la transparencia.

Según la definición de López et al. (2005:40) un cibermedio es "aquel emisor de contenidos que tiene voluntad de mediación entre hechos y público, utiliza fundamentalmente criterios y técnicas periodísticas, usa el lenguaje multimedia, es interactivo e hipertextual, se actualiza y se publica en la red Internet". Sin embargo, una observación preliminar apunta que no todos cumplen las premisas. La hipótesis que se plantea es que los nuevos medios digitales presentan déficits en algunos aspectos estructurales, de contenido y relacionados con la responsabilidad editorial. De igual modo, partimos de que prácticas y recursos habituales en los medios digitales de ámbito nacional (Fondevila, 2014) no se han incorporado a estos medios de proximidad, estancados en la mayoría de los casos en diseño e innovación.

Esta propuesta no pretende presentar una jerarquización o una categorización de los medios en función de un índice de calidad pero sí posibilita revisar el comportamiento de las variables, extraer algunos patrones $\mathrm{y}$, a través de la medición horizontal, identificar aquellos medios que puedan erigirse en referentes en el entorno local y regional. Esta investigación constituiría así un punto de partida para futuros estudios de contenido en torno a variables clásicas que afectan a la calidad de la información - criterios de selección y agenda, equilibro temático, variedad de fuentes - y de las noticias - estilo narrativo, género periodístico, contextualización -, todos ellos recogidos en el reconocido sistema VAP (Valor Agregado Periodístico) desarrollado por la Universidad Católica de Chile (Alessandri et al., 2011) y 
que se podría aplicar a una muestra más reducida de medios.

\section{EL CONCEPTO DE CALIDAD EN LOS MEDIOS DE COMUNICACIÓN}

La primera dificultad, previa al diseño de indicadores, es tratar de lograr una definición consensuada de la calidad aplicada a un bien intangible, la información, pero que se transmite a través de productos materiales, los medios. Suele ser más sencillo identificar la falta de calidad o aquello que debiera ser evitable (Picard, 2004) que definir unos estándares de corrección y buen hacer. Las propuestas metodológicas de carácter empírico para medir la calidad se han localizado, sobre todo, en la academia alemana y en sudamericana. Schulz (2000) apunta que la calidad está relacionada con tres factores: la disponibilidad de recursos, un ordenamiento político garante de la libertad de actuación de los medios y, en tercer lugar, los mecanismos de autorregulación. De ahí, que la calidad no pueda ser considerada un concepto unívoco. Por ejemplo, la propuesta de Gutiérrez Coba (2006) distingue entre calidad informativa y calidad periodística, ya que los elementos que componen a esta última afectan a todo el proceso de producción, no sólo al producto final.

Entendemos que la calidad tiene que ver con el volumen de producción original del medio (Picard, 2004) pero también debe ser estudiada en su contexto (Gómez Mompart y Palau, 2013) y puede estar relacionada con elementos consustanciales al medio, no sólo con la información. En este sentido, coincidimos con De Pablos y Mateos en que es necesario "aclarar al lector lo que se le ofrece como periodismo" (2004:356), con una política de transparencia que permita conocer aspectos como los principios editoriales del medio, las condiciones de trabajo de los periodistas o los datos relativos a la dimensión empresarial, todo ello exigible, máxime cuando perciben dinero público a través de las administraciones. Este trabajo encuentra su base en la calidad vinculada a la responsabilidad social de los medios (De la Torre y Téramo, 2015).

En lo que respecta al soporte, el medio digital presenta unas características propias entre las que destaca la hipertextualidad, la interactividad, la multimedialidad y la memoria (Díaz Noci, 2008), que también afectan a la calidad periodística. Por otro lado, es obvio que aspectos técnicos como el diseño, la interfaz o la correcta colocación de elementos son fuentes de sentido y determinan tanto el acceso a la información, la jerarquía informativa y el proceso de interpretación. Como base de este estudio se han revisado diversas propuestas analíticas, que toman como base la observación externa de los medios. El investigador Lluís Codina reúne varios trabajos sobre calidad y diseño de los cibermedios, así como metodologías específicas de análisis, entre los que destacamos por su utilidad, la ficha publicada en 2011 en el volumen Ferramentas para Análise de Qualidade no Ciberjornalismo. Uno de los trabajos más recientes es el de Romero Rodríguez et al. (2016). Los investigadores formulan una taxonomía de 75 dimensiones agrupadas en tres macroáreas que agrupan aspectos empresariales, sociolaborales y de contenido, aunque para determinar el cumplimiento de algunas de ellas es necesaria la indagación en el seno del medio o bien su compromiso de respuesta veraz, y no sólo un análisis de lo que se presenta accesible a través de la observación externa.

Y en todo este proceso de definición de calidad, cabe preguntarse: ¿qué papel juega la audiencia? Creemos relevante, a la hora de fijar los estándares de medición sobre la calidad, tomar en consideración el contexto y las características de los receptores potenciales. El $32,7 \%$ de la población de Castilla-La Mancha se sitúa en los segmentos de clase media baja o baja y es la tercera comunidad autónoma que presenta el índice socioeconómico más bajo de 
España (AIMC, 2017). En lo que respecta al nivel educativo, el 69,4\% carece de estudios superiores, siendo sólo un 16,4\% los que cuentan con nivel universitario. El porcentaje de población sin ningún tipo de estudios o con Educación Primaria es del 26,7\% (CIS, 2015). La frágil estructura mediática que presenta respondería a una sociedad informativamente deprimida y dominada por el consumo audiovisual (Díaz Nosty, 2005), lo que genera cierta incertidumbre sobre cómo se produce la interacción entre medios y ciudadanía y qué valor otorga a la calidad cada una de estas instancias. A la luz de un contexto sociocultural como el descrito, sería relevante indagar qué peso tienen, tanto en la producción como en el consumo mediático, criterios como la originalidad en la agenda temática, la variedad de fuentes o elementos innovadores de diseño. Es factible concluir que este entorno exige, con mayor ahínco, establecer sistemas de promoción de la calidad que favorezcan un consumo crítico y una mejor comprensión de la información.

En este sentido, uno de los hitos en relación a políticas públicas destinadas a mejorar la calidad de los medios informativos regionales lo podemos situar en el Acuerdo Marco impulsado por la Junta de Comunidades de Castilla-La Mancha en septiembre de 2016 para modificar el sistema de reparto de la publicidad institucional de esta administración. Según la nueva normativa para "la racionalización, ordenación y adopción de los tipos contratables relativos a la compra de espacios publicitarios con empresas titulares de medios comunicación e información, locales, comarcales, provinciales y regionales" (JCCM, 2016), los medios que quieran optar a contratantes deben acreditar:

a) Solvencia técnica/profesional demostrada mediante la declaración del personal contratado, con jornada completa o parcial, y en todo caso debe aparecer como mínimo un periodista con la acreditación correspondiente.

b) Estar sometidos a un sistema de control de audiencia, bien OJD Interactiva o ComScore.

Se trata de la primera iniciativa para establecer criterios mínimos transparentes en la distribución de las campañas, si bien no aplica una valoración cualitativa a los solicitantes, cuestión que sí recoge la mayoría de convocatorias autonómicas en otras comunidades cuando hablamos de ayudas públicas a medios de comunicación (Galletero y López Cepeda, 2018). Siguiendo los criterios aprobados, durante 2016 recibieron contratos publicitarios 32 medios digitales de la región, por un montante total de 708.749 euros.

\section{METODOLOGÍA}

Este estudio forma parte de una investigación más amplia llevada a cabo en el Observatorio Mediacom y adopta una metodología combinada cualitativa y cuantitativa. A partir del primer mapeo de digitales definido en 2015 se han realizado seguimientos anuales en 2016 y 2017 para registrar altas y bajas en el censo de medios digitales y detectar cambios sustanciales en las plataformas de publicación. A lo largo de 2017, se ha desarrollado una etnografía virtual (Hine, 2004) y la consulta mensual de los digitales con mayor circulación en la región ha permitido identificar cuáles son los elementos estructurales, de diseño y calidad informativa que definen los estándares más comunes entre los medios regionales. Con esta base se han identificado los ítems para diseñar una plantilla sistemática de análisis y un libro de códigos con el fin de obtener resultados objetivables. No es inusual que en el marco de los observatorios se construyan modelos adaptados (Aguirre y Bernal, 2014) ya que la abstracción de la calidad como concepto puede dificultar el establecimiento de métricas comunes. Adoptar un modelo basado en la excelencia (Rodríguez- Martínez et al., 2012) 
carecería de eficacia comparativa si la mayoría de los indicadores dan como resultado incumplimiento. Por esta razón, se propone el diseño de una serie indicadores adaptados al contexto de referencia regional. En este análisis, de acuerdo a la técnica de la observación externa, el punto de vista que adopta el investigador es el del usuario, tomando como unidad de análisis el medio digital como producto informativo en su versión de escritorio. No obviamos que el consumo mediático actual se produce a través de múltiples dispositivos, principalmente el móvil, pero en el caso de nuestra muestra no todos los medios cuentan con una versión adaptada, como veremos. Para medir los ítems relacionados con la transparencia, se han tomado en consideración los datos de acceso público que ofrece la web y para el resto de apartados se ha considerado también la portada como la interfaz más completa de relación con el lector. Las mediciones se efectuaron entre los meses de septiembre y octubre de 2017. La recogida de información de la muestra se efectuó de acuerdo a un proceso de intercodificación (Mayntz et al., 1993), validado mediante un libro de códigos compartido por ambas observadoras, para considerar o no el cumplimiento en cada uno de los ítems.

\subsection{Corpus}

Para definir la muestra se ha tomado como base el censo de los 63 medios digitales nativos y sobre él se han establecido dos criterios de selección. En primer lugar, se han considerado aquellos que percibieron en 2016 fondos públicos por parte de la Junta de Comunidades a través de contratos de publicidad institucional (32 medios).

Ilustración 2: Importes abonados por la administración regional a los medios digitales de la muestra en concepto de publicidad institucional durante el año 2016

\begin{tabular}{|l|l|}
\hline MEDIO DIGITAL & IMPORTE \\
\hline EnCastillalamancha.es & 104.696 \\
\hline La Cerca & 88.107 \\
\hline El Día Digital & 50.006 \\
\hline El Digital de C-LM & 45.721 \\
\hline El Digital de Albacete & 45.498 \\
\hline Periódico CLM & 30.925 \\
\hline DCLM.es & 30.502 \\
\hline Voces de cuenca & 27.300 \\
\hline CLM 24 & 23.961 \\
\hline La Comarca de Puertollano & 16.264 \\
\hline El Semanal de la Mancha & 14.131 \\
\hline Mancha Información & 10.717 \\
\hline Guadalajara Diario & 9.850 \\
\hline La Crónica de Guadalajara & 9.670 \\
\hline Albacete abierto & 6.568 \\
\hline Albacete capital & 6.398 \\
\hline Cuenca News & 5.609 \\
\hline Tarancón Digital & 4.690 \\
\hline Web Toledo & 3.623 \\
\hline ValdeRec & 2.372 \\
\hline
\end{tabular}

Fuente: Portal de Transparencia de la Junta de Comunidades de Castilla-La Mancha 
Esta acotación responde a que cabría exigir un ejercicio mayor de rendición de cuentas y transparencia (Mauri y Ramón, 2015) a los medios perceptores de fondos públicos. De ellos, se han extraído los que en el primer semestre de 2017 han sido sometidos a una medición de visitas estable y regular por parte de la auditoría OJD Interactiva, puesto que puede considerarse un indicador de la profesionalidad del medio y de su vocación de permanencia. Precisamente y fruto de la exigencia de la administración regional de contratar sistemas de medición fidedignos, el número de medios que han solicitado el control de OJD ha ido incrementándose a lo largo de 2016, siendo sólo dos los registrados con anterioridad a septiembre de 2016 y veintiuno a comienzos de 2017. De los 21, se ha excluido de la muestra la edición regional de eldiario.es ya que su dependencia de la edición nacional en recursos y contenidos desvía los resultados. El conjunto de medios que componen el corpus (20) representa un $76 \%$ del presupuesto destinado por la Junta a publicidad en medios digitales, esto es, 538.715 euros.

Todos ellos son de titularidad privada y su distribución geográfica es la siguiente: 3 corresponden a la provincia de Albacete, 3 a Cuenca, 3 a Guadalajara, 4 a Ciudad Real, 1 a Toledo y 10 son de ámbito regional.

\subsection{Ficha de análisis}

La propuesta de análisis contempla las siguientes dimensiones:

a) En el indicador de transparencia se ha observado si aparecían en la web del medio los siguientes datos: nombre de la empresa editora; tipo de sociedad; quién es el propietario o socio mayoritario; identificación del director/a; del redactor/a jefe; información sobre la estructura de la plantilla; información sobre principios editoriales, no sólo en términos ideológicos, sino de definición del área temática o valores informativos prioritarios; información sobre la situación financiera del medio (principales anunciantes, suscriptores, subvenciones recibidas); datos sobre la medición de audiencias o, al menos, información sobre a qué tipo de medición está sometido el medio.

b) En el indicador de redes sociales se ha buscado valorar el grado de adaptación del medio a la web 2.0. Para ello, se han registrado todas las redes disponibles de cada medio; su número de seguidores o suscriptores en las más importantes (Twitter, Facebook y YouTube); si han incorporado otras redes sociales más novedosas para compartir contenido como Whatsapp, Instagram o Snapchat; si cuentan con una aplicación móvil y si incluyen blogs.

c) Por último, el indicador de diseño y estructura engloba cinco parámetros:

1) Navegabilidad - Cabecera identificada con claridad; menú con secciones reconocibles y estables; uso de etiquetas de navegación y diseño adaptado al formato móvil.

2) Acceso a la información - Buscador por palabras; sección "Lo más visto/leído"; sección de servicios; sección "Última hora"; hemeroteca y contenido de opinión correctamente identificado.

3) Multimedia: Sección específica de videos, galerías de fotos y retransmisiones en directo.

4) Interacción: Comentarios habilitados, sistema de votación de los artículos, encuestas e incorporación del contenido del usuario.

5) Personalización: Herramientas de sindicación de contenidos, boletines electrónicos y registro de usuarios para personalización de la web.

Una última parte de la ficha estaba destinada al análisis cualitativo para facilitar la correcta identificación y valoración de los ítems por parte de las investigadoras, y para explorar el predominio de noticias de portada con una firma identificable o de informaciones firmadas 
por la redacción o por agencias. En este apartado se han valorado, además, los problemas concretos de diseño como exceso de publicidad, falta de armonía en los menús, espacios en blanco injustificados... Estos análisis de orientación cualitativa han facilitado la identificación de medios en los que domina la mala o la buena praxis.

\section{RESULTADOS}

\subsection{Indicador de transparencia}

Los resultados confirman que la transparencia es uno de los aspectos más deficientes en los medios digitales de Castilla-La Mancha. Aunque a priori los recursos de la web 2.0 podrían favorecer la aparición de nuevos sistemas de supervisión y transparencia (Mauri y Ramón, 2015), en los cibermedios objeto de análisis no sólo se detecta la carencia de los más novedosos - botón de notificación de errores, blog del director, chats con los lectores, entre otros - sino de aquellos clásicos que permiten obtener información básica como la identificación del director o la declaración de los principios editoriales. La mayor parte de los medios presentan un alto incumplimiento de los diez criterios observados en este indicador. Sólo Guadalajara Diario cumple nueve de ellos, siendo el único medio que ofrece la información acerca de quiénes son los propietarios de la sociedad editora. Sólo un 38\% de los medios superan cinco requisitos cumplidos, siendo mayoría los que se sitúan entre tres y cuatro ítems. ValdeRec es el caso más evidente de falta de transparencia: no cumple ningún ítem del indicador y, además, el aviso legal que mantiene corresponde a otra empresa. Le sigue como medio menos transparente WebToledo, que sólo presenta el nombre del director y una vía de contacto con la redacción, ítem este último, junto al nombre de la sociedad editora, que figura en mayor número de medios. Con respecto al tipo de sociedad, la mayoría de las identificadas (15) son Sociedades Limitadas, una Sociedad Anónima (El Día Digital) y una entidad cooperativa (Albacete Abierto).

Ilustración 3: Número de medios que cumplen los indicadores de transparencia

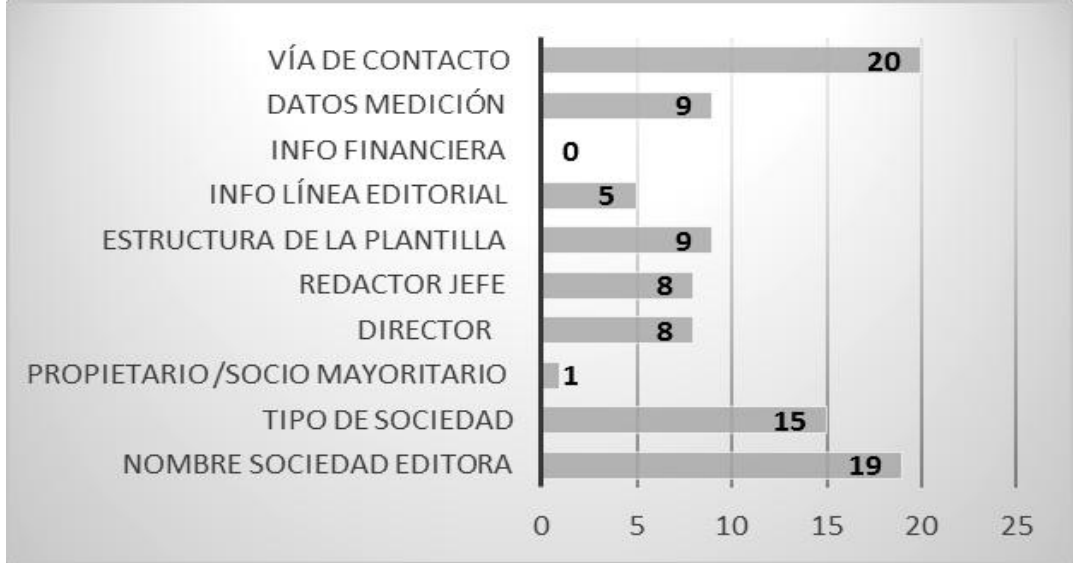

Fuente: Elaboración propia

Resulta preocupante que sólo 7 de los 21 medios de la muestra ofrezca el nombre del director e informe sobre la composición de la plantilla, un dato que permitiría saber si son 
profesionales de la información cualificados quienes están elaborando el contenido o, al menos, cuántos son. En el caso de La Crónica de Guadalajara, en el apartado 'Quiénes somos' se ofrece un formulario para solicitar información. Por último, sólo 5 medios (Albacete Capital, Guadalajara Diario, El Digital de C-LM, EnCastillalamancha.es y La Cerca) ofrecen a sus lectores una mínima aproximación a su línea editorial, aunque en el caso de La Cerca únicamente ofrece noticias de los premios obtenidos.

\subsection{Indicador de redes sociales y web 2.0}

Una de las vías de acceso a las cuentas de las redes sociales de un medio suele ser su portada, por ello para la toma de datos de este indicador se han contabilizado aquellas presentes en la página principal de cada medio. En este sentido, hay dos medios que mantienen cuentas en redes sociales pero no las enlazan desde su portada, Albacete Capital y La Crónica de Guadalajara, siendo este último caso el más llamativo al tratarse de uno de los digitales pioneros en la región, con origen en 1999. Sólo un medio, de nuevo WebToledo, carece de perfil alguno. En el otro extremo, se encuentra Henares al Día con perfiles en cinco redes sociales, el único medio de los analizados que se ha incorporado a Instagram, una de las redes que presenta un mayor crecimiento, sobre todo entre los sectores más jóvenes de la población. Será importante monitorizar el proceso de incorporación a esta red del resto de medios digitales castellano-manchegos para detectar cuáles son las estrategias de conexión con las nuevas generaciones de lectores que marcan las pautas del consumo informativo.

Ilustración 4: Presencia en redes sociales

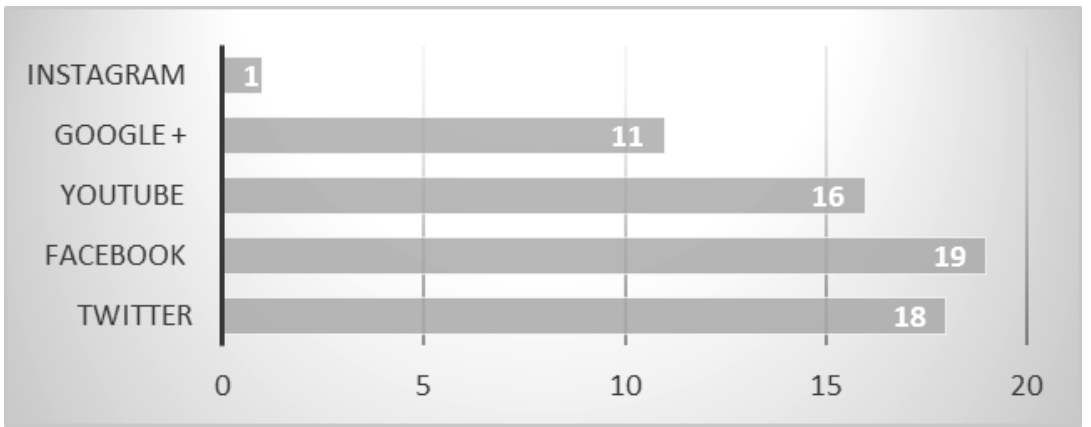

Fuente: Elaboración propia

Sobre el número de seguidores, se observa que el impacto en redes en términos cuantitativos no siempre se corresponde con la calidad, entendida en términos de producción de información propia y exclusiva. Así, el medio líder en Twitter y Facebook es CLM24 y su éxito se debe a que funciona como un repositorio de las noticias de agencia sobre Castilla-La Mancha, piezas que replica sin reelaboración tanto en su web como en todas sus redes. En términos generales, los medios de la muestra no tienen un alto impacto en Twitter, tras CLM24 (56,6 k) el siguiente medio en el ranking de seguidores es La Cerca, que desciende hasta los 28,5 k, mientras que un tercio de los medios de la muestra están por debajo de los 4.000 seguidores. En Facebook, las diferencias son aún mayores, en primer lugar vuelve a situarse CLM24 con más de 129.000 seguidores; en segunda posición está El Digital de Albacete con 88.323, mientras que 13 de los 20 medios con cuenta en esta red no superan los 9 mil seguidores.

Destaca el hecho de que los medios no hayan incorporado ningún elemento innovador 
referido a la difusión de contenido en el móvil, como un canal de Whatsapp o de Telegram. Es reseñable el uso de ISSUU por parte de Cuenca News como plataforma para subir documentos (programas de fiestas, clasificaciones deportivas) aunque se presenta sin actualización regular. Lo mismo ocurre con otros enlaces residuales a cuentas sin uso que no han sido dadas de baja y permanecen en las portadas como sucede en Cuenca News o Tarancon Digital (Myspace, Flickr o Linkedin). Estas cuentas muertas que permanecen visibles son ejemplos de malas praxis en la gestión de redes. Es generalizado el uso de YouTube aunque el número de suscriptores es muy bajo excepto en Cuenca News, La Cerca y El Digital de Albacete (estos dos últimos superan las 2.000 suscripciones). Estos medios son, al mismo tiempo, los que generan contenido audiovisual original. La Cerca y Voces de Cuenca mantienen, además, una cuenta en Vimeo. Sólo el 24\% hace uso de blogs. Ningún medio señala en su web la posibilidad de descargar su aplicación para móvil, aunque dos sí la tienen: Albacete Capital y El Digital de Albacete.

\subsection{Indicador de diseño y estructura}

1) Navegabilidad: Se confirma la poca originalidad en la estructuración de las páginas web, con portadas que mantienen las secciones tradicionales de la prensa impresa, reduciendo las posibilidades de una navegación más intuitiva. O bien medios que sitúan al mismo nivel las secciones fijas con aquellas coyunturales que remiten, por ejemplo, a la celebración de una fiesta. En aquellos de ámbito regional la distribución de la información se organiza también por áreas geográficas, aunque no mantienen corresponsalías en las distintas provincias, lo que apunta a informaciones procedentes, en su mayoría, de comunicados de prensa y agencias. Los medios digitales en Castilla-La Mancha mantienen una fuerte dependencia de la publicidad institucional y de la local procedente del sector privado, lo que provoca que se incluyan numerosos banners de tamaño pequeño y mediano, no siempre respetando las reglas del diseño gráfico para favorecer la legibilidad. En algún caso la cabecera queda desdibujada ante la proporción de publicidad y la posición preferente del banner superior (Tarancón Digital).

Ilustración 5: Portada de Tarancón Digital

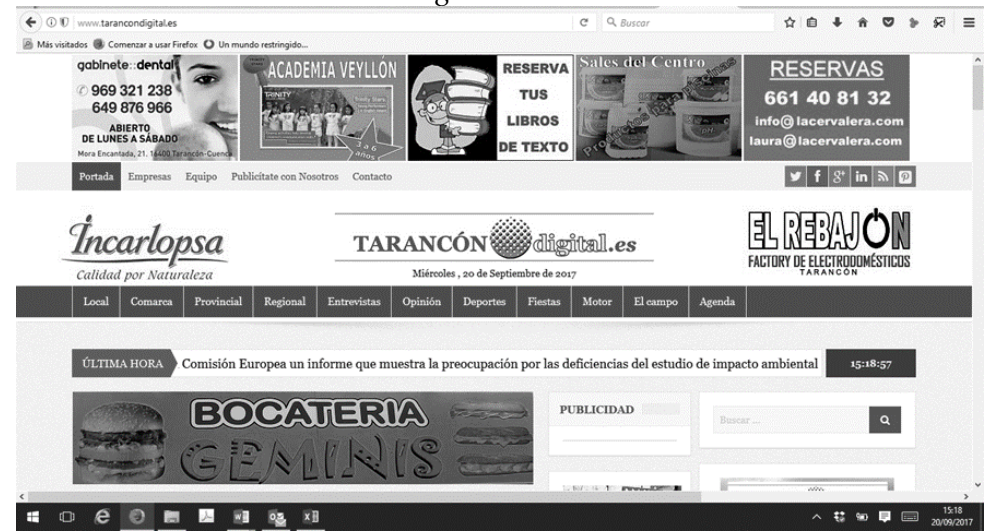

Fuente: www.tarancondigital.es (20/09/2017)

En el análisis de la muestra, además de una tendencia a la desorganización en la colocación de elementos en la interfaz, se han localizado secciones vacías en El Día digital 
('Nosotros') y en Web Toledo algunas secciones temáticas que en apariencia son del medio (España, Religión...) remiten a páginas externas (El Mundo, Aciprensa, Diez Minutos, ABC $C-L M)$ o presentan enlaces que no funcionan ('Farmacias de guardia'). En La comarca de Puertollano las secciones aparecen únicamente en el apartado destinado a Hemeroteca, sin que exista un menú temático.

Las etiquetas permiten representar los contenidos y constituyen puntos de interacción (Acosta et al., 2011) por lo que se interpretan como un indicio de mayor navegabilidad. Este recurso se utiliza tan sólo en 12 medios y su uso se relaciona con las categorías de las secciones (Provincias, Deportes, Región...) por lo que no facilitan la relación temática, ni permiten el seguimiento transversal de las informaciones. Incluso se localiza algún caso (CLM24) en el que están generadas de manera automática reproduciendo las palabras del titular, de tal modo que la etiqueta puede no tener contenido semántico ("de", "se") ni utilidad como herramienta orientativa o de contexto ("considera", "factible"). Otro aspecto reseñable es que sólo el $23 \%$ hace uso del diseño web adaptativo entendido como una técnica de diseño y desarrollo web que consigue adaptar el sitio web al entorno del usuario (Labrada y Salgado, 2013), que puede ser un dispositivo móvil o una tableta. La observación evidencia pocos recursos para mejorar la experiencia de navegación: predomina en los medios el texto plano, sin hipervínculos, sin epígrafes, sin ladillos, ni negritas. Se replican las rutinas de redacción de los medios impresos sin aprovechar las potencialidades del medio.

2) Acceso a la información: En este apartado se han localizado aquellas herramientas que tienen como fin facilitar al usuario el acceso al contenido deseado. El uso de este tipo de recursos es claramente minoritario: sólo 9 medios tienen una sección denominada 'Hemeroteca', aunque en 3 de ellos el sistema corresponde a un buscador por palabras. Este último elemento, que permite la búsqueda por palabra clave, es el más estandarizado, localizado en el $76 \%$ de los medios. Otro resultado concluyente es la desaparición de la información de servicios, ofrecida sólo por 9 medios y, en algunos casos, presentada de manera desordenada y desactualizada.

Ilustración 6: Medios que cumplen los indicadores de acceso a la información.

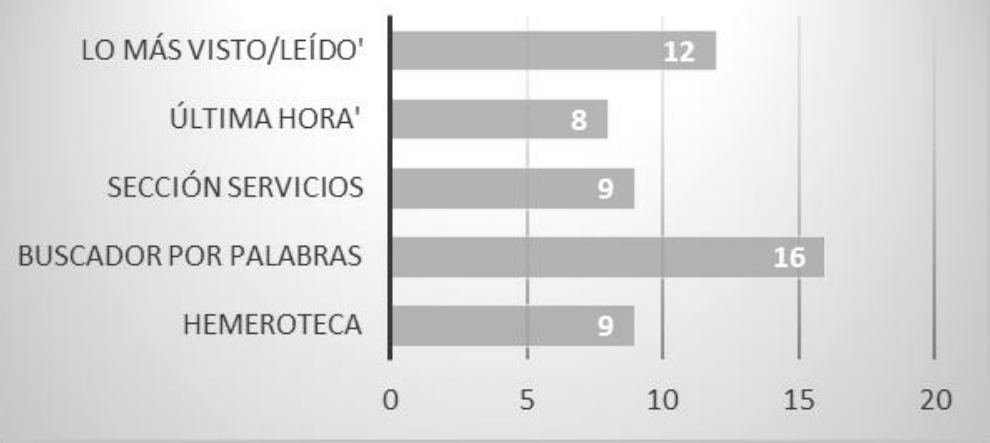

Fuente: Elaboración propia

Los apartados de 'Última hora' y 'Lo más visto/leído' también cuentan con baja implantación. El Semanal de la Mancha además de 'Lo más visto', tiene 'Lo más guardado' y 'Lo más comentado'; sin embargo, se da la paradoja de que no existen comentarios en ninguna de las noticias de este ranking. La crónica de Guadalajara incluye en portada una 
nube de etiquetas para señalar los temas destacados, pero no se identifica si esta selección corresponde a lo consultado por los lectores o a decisiones editoriales.

Prácticamente todos los medios analizados (19) ofrecen contenidos de opinión, bien en forma de artículos o blogs personales; por norma general, separan la información de la sección de opinión. Sin embargo, hemos encontramos algunos problemas producidos por el manejo de las plantillas de edición, donde el nombre del editor se confunde con la firma del articulista (ValdeRec).

3) Contenido multimedia: El uso de la información audiovisual es muy reducido, con sólo 5 medios que difunden vídeos de elaboración propia y otros 3 que difunden piezas audiovisuales procedentes de plataformas o instituciones. El Digital de Albacete y La Cerca son aquellos que más han incorporado la multimedialidad, realizando también retransmisiones en directo. Aunque más de la mitad de los digitales cuenta con una sección para ofrecer una galería fotográfica, es frecuente que las fotografías aparezcan sin firma e incluso sin pie de foto explicativo, lo que indicaría una devaluación de la información gráfica como elemento periodístico. Sólo uno de los medios ha integrado el formato sonoro, $\mathrm{La}$ Crónica de Guadalajara en su sección 'El sonido de la noticia', aplicando el formato de una noticia radiofónica. En términos de innovación cabe destacar la iniciativa de Voces de Cuenca que retransmite los plenos del Ayuntamiento a través de titulares en Twitter.

4) Interacción y personalización: Una amplia mayoría suspende en la interacción a través del portal, aunque podemos suponer que parte de esta interacción sí se produce a través de las redes sociales. Todavía un tercio de los medios estudiados carece de sistema para comentar las noticias y en los que tienen los comentarios activados no se observa apenas participación. Los sistemas de votación sólo están habilitados en 5. Las encuestas se localizan en 3 digitales y se plantean de manera esporádica. La incorporación de contenidos del usuario está limitada, al contar sólo 8 con alguna vía para enviar información que pueda ser publicada, si bien no siempre esta sección está actualizada como sucede con 'Fotografías de los lectores' de El Día digital, cuyas últimas publicaciones son de 2015.

El último aspecto valorado ha sido la posibilidad de personalizar el contenido a través de sistemas que permiten al usuario preseleccionar los contenidos a su gusto. De los 21 medios, 12 ofrecen sindicación de contenidos, en ocasiones con algún grado de personalización (Periódico CLM y Cuenca News), 10 la posibilidad de suscripción al boletín electrónico y 3 cuentan con registro de usuarios. Interpretamos que todos estos sistemas, que presentaron cierta utilidad e innovación en sus inicios a finales de los 90 (Franganillo y Catalán, 2005), han sido sustituidos por las redes sociales como instrumentos para canalizar las actualizaciones de la información en los sites.

La combinación de la metodología cuantitativa y cualitativa permite concluir que los medios que alcanzan mayor grado de cumplimiento de los ítems en los cinco parámetros que afectan al contenido son Cuenca News, CLM24, La Cerca y Voces de Cuenca.

\section{CONCLUSIONES}

En primer lugar, es conveniente destacar que la investigación que lleva a cabo el Observatorio MediaCom exige un seguimiento continuado, dado que se realiza en un contexto altamente dinámico. Los medios digitales están en constante transformación y 
adaptación, por ello estos resultados ofrecen una foto fija de un proceso, un mapa de situación que será más útil en la medida en que se repliquen las mediciones de manera sistematizada en un plazo próximo.

El valor de esta contribución reside, por tanto, no sólo en los resultados sino en el establecimiento de una metodología de análisis. El diseño de indicadores adaptados a los contextos particulares como Castilla-La Mancha es importante, porque solo sobre la base de lo que opera en el territorio se pueden ofrecer resultados analíticos potencialmente transferibles que sean de utilidad tanto a los propios medios como a las administraciones públicas, que están formulando nuevos modelos de financiación y distribución de la publicidad institucional. Se trata de una cuestión relevante, pues la observación de los medios, y la publicidad inserta en ellos, revela una fuerte dependencia de los recursos públicos.

En relación a la producción de indicadores, la exploración realizada nos permite cuestionar que los medidores cuantitativos tradicionales (número de visitas, seguidores en redes) sean suficientes para funcionar como criterio exclusivo de captación de inversión publicitaria (sea pública o privada), ya que estas cifras no siempre se corresponden con la calidad, la innovación y el volumen de producción propia de los medios. Con respecto a los contenidos informativos, una tendencia compartida es la baja producción propia $\mathrm{y}$, al contrario, la excesiva publicación - sin modificaciones - de notas de prensa, comunicados y noticias de agencia. Un indicio es la falta de firma, los créditos de las noticias de portada suelen atribuirse a la redacción o a la agencia.

$\mathrm{Si}$ atendemos a los indicadores de transparencia, diseño y estructura es notable el margen de mejora que presentan los digitales castellanomanchegos. Para fomentar un consumo crítico de los medios y en línea con los principios de la cultura de transparencia, debería incrementarse la información sobre el medio que está disponible para los lectores, al menos acerca de las estructuras de las plantillas, principios editoriales y composición de la sociedad editora. En sintonía, las administraciones que realizan aportaciones económicas a los medios mediante publicidad institucional (Junta de Comunidades, diputaciones y ayuntamientos) podrían ser cada vez más ambiciosas en relación a la concesión de los contratos publicitarios, exigiendo una serie de parámetros que animen a la modernización y mejora de los digitales. El número de visitas es, a día de hoy, el principal indicativo para fijar las cuantías de la publicidad, tanto pública como privada, y no se han implementado aún sistemas que tengan en cuenta otro tipo de indicadores. Si bien el estudio expuesto es de carácter descriptivo, hemos apreciado cierta relación entre los medios que cumplen menos ítems y los que percibieron importes menores por parte de la JCCM. Sin embargo, no hay forma de saber qué impacto tienen los ingresos públicos en los procesos de mejora de los digitales regionales y creemos que un sistema de indicadores que permita un seguimiento monitorizado facilita una primera evaluación.

En general, los medios carecen de propuestas originales e innovadores y se observa que no están aprovechando, en la mayoría de los casos, las potencialidades del medio digital. Un modelo interesante como ejemplo de buenas prácticas es EnCastilla-La Mancha.es, un digital en el que se aprecian indicios de calidad como mayor volumen de contenido original, uso de hipervínculos y sumarios de mayor tamaño que facilitan la legibilidad, e identificación de la autoría en las noticias. La web señala que su rediseño ha estado cofinanciado por el Fondo Europeo de Desarrollo Regional para hacer una página SEO friendly, cualidad que alude a su mejor posicionamiento web. El Digital de Albacete destaca por las innovaciones incorporadas - aplicación móvil, canal de YouTube actualizado y con alto grado de seguimiento - pero presenta algunas deficiencias de diseño como una estructura de secciones 
poco ordenada y un exceso de publicidad, que impide distinguir con claridad el titular y lo separa del cuerpo de la información. Por el contrario, WebToledo constituye un ejemplo de medio no profesionalizado, con un diseño desestructurado e inadaptado al lenguaje 2.0 y sin incorporación apenas de los indicadores observados. El digital de Valdepeñas ValdeRec destaca por su acusada falta de transparencia.

Por último, es oportuno reseñar que los recursos que se han considerado en este análisis no requieren grandes inversiones. Tampoco se proponen medidas excesivamente ambiciosas. Consideramos, por ello, que, a partir de un buen diagnóstico, su implantación está al alcance de, si no todos, la mayoría de los medios digitales. Este trabajo proporciona una orientación, basada en los criterios de la academia sobre calidad, sobre cuáles son los medios de referencia en la región en los citados aspectos, con el fin de diferenciar y apoyar a aquellos que funcionan de una manera más profesionalizada.

\section{BIBLIOGRAFÍA}

Acosta, M.; Ramírez-Céspedes, Z. y Marrero, L. (2011) “Organización de la información en sitios web periodísticos". El profesional de la información, 20 (1): 54-60, DOI: https://doi.org/10.3145/epi.2011.ene.07

Aguirre, C. y Bernal, J. D. (2014) "Contenidos periodísticos digitales: hacia un modelo de medición de calidad”, Revista ComHumanitas, 5 (1): 75-90. https://goo.gl/G9yc71_[consulta 5/8/2018].

Alessandri, F. et al. (2011) "VAP: un sistema métrico de la calidad periodística". Cuadernos.Info, 14: 112-120, DOI: https://doi.org/10.7764/cdi.14.187

Asociación para la Investigación de Medios de Comunicación (2007-2017) Marco General de los Medios en España. Ediciones anuales.

Centro de Investigaciones Sociológicas (2015) Estudio $n^{\circ} 3093$ Postelectoral Elecciones Autonómicas Castilla-La Mancha. http://datos.cis.es/pdf/Es3093mar_A.pdf [consulta 16/01/2018].

Codina et al. (2011) "Ferramenta para Análise Geral de Qualidade em Cibermeios". En M. Palacios, (Org.) Ferramentas para Análise de Qualidade no Ciberjornalismo. Covilhã, Portugal: Livros LabCom. pp. 21-35.

Cruz, J. y Suárez-Villegas, J. C. (2017) "Pautas deontológicas para el periodismo digital”. El profesional de la información, 26 (2): 249-254.

De Bustos, J. y Casado, M. (2010) "Momentos de crisis en la industria periodística". TELOS, 83: 24-35.

De La Torre, L. y Téramo, M. T. (2015) "La calidad de la información periodística: estrategias para su observación. Coincidencias y divergencias entre los medios y el público". Estudios sobre el Mensaje Periodístico, 21: 135-144. https://goo.gl/BhrQ3T [consulta 2/10/2017].

De Mateo, R; Bergés, L. y Garnatxe, A. (2010) "Crisis, ¿qué crisis? Los medios de comunicación: empresas y periodismo en tiempos de crisis". En F. Campos Freire (Coord.) El cambio mediático. Zamora: Comunicación Social ediciones y publicaciones, pp.75-106.

De Pablos, J.M. y Mateos, C. (2004) "Estrategias informativas para acceder a un periodismo de calidad, en prensa y TV". Ámbitos, 11-12: 341-365.

Dewey, J. (2004) El público y sus problemas. Madrid: Ediciones Morata.

Díaz Noci, J. (2008) "Definición teórica de las características del ciberperiodismo: elementos de la comunicación digital”. Doxa Comunicación, 6: 53-91. https://goo.gl/KcBHwJ [consulta 1/7/2018].

Díaz Nosty, B. (2013) La prensa en el nuevo ecosistema informativo. ;Que paren las rotativas! Madrid: Fundación Telefónica.

Díaz Revorio, F. J. (2002) "La situación de los medios de comunicación social". En Castilla-La Mancha" en Cortes de Castilla-La Mancha - Universidad de Castilla-La Mancha (Ed.) Parlamento y Constitución. Anuario 2002, 6: 255-272. https://goo.gl/eXhzJ5 [consulta 15/09/2018].

Fondevila, J. F. (2014) "El uso de hipertexto, multimedia e interactividad en periodismo digital: propuesta metodológica de ranking de calidad". ZER, 19 (36): 55-76. https://goo.gl/rxKBa5 
[consulta 4/6/2018].

Franganillo, J. y Catalán, M. A. (2005) "Bitácoras y sindicación de contenidos: dos herramientas para difundir información", Textos universitaris de biblioteconomia $i$ documentació, 15. https://goo.gl/CBB3MD [consulta 2/10/2017].

Galletero, B. (2018) Del periódico impreso al diario digital: Estudio de una transición en Castilla-La Mancha (tesis doctoral). Cuenca: Universidad de Castilla-La Mancha.

Galletero, B. y López Cepeda, A. M. (2018) "Ayudas directas y publicidad institucional a medios de comunicación en el escenario autonómico: revisión de indicadores y pautas de mejora". El profesional de la información, v. 27 (3): 682-691.

Gómez-Mompart, J.L y Palau, D. (2013) "El reto de la excelencia. Indicadores para medir la calidad periodística”. En J. L. Gómez-Mompart; J. F. Gutiérrez Lozano y D. Palau, (Eds.) La calidad periodística. Teorías, investigaciones y sugerencias profesionales. Barcelona: Servei de Publicacions de la Universitat Autònoma de Barcelona, pp.17-38.

Gutiérrez Coba, L. (2006) "Análisis de la calidad informativa, primer paso hacia el cambio". Palabra Clave, 9 (1): 29-56. http://www.redalyc.org/html/649/64900102/ [consulta 2/10/2017].

Hine, C. (2004) Etnografía virtual. Barcelona: Editorial UOC.

JCCM (2016) Pliego que rige el Acuerdo Marco para la racionalización, ordenación y adopción de los tipos contratables relativos a la compra de espacios publicitarios. Toledo: Secretaría General de la Presidencia, accesible en: https://goo.gl/1HTqKY [consulta 25/11/2017]

Labrada, E. y Salgado, C. (2013) "Diseño Web Adaptativo o Responsivo". Revista Digital Universitaria, 14 (1). https://goo.gl/HVk1zo [consulta 20/2/2018].

Laguna, A. et al. (2016) Los medios de comunicación en Castilla-La Mancha. Informe 2015 Mediacom. Servicio publicaciones UCLM, DOI: http://dx.doi.org/10.18239/ins_117.2016.01

López Villaverde, A. L. y Sánchez, I. (1998) Historia y evolución de la prensa conquense (1811-1939). Cuenca: Servicio de Publicaciones de la Universidad de Castilla-La Mancha.

López, X. et al. (2005) "Tipología de los cibermedios". En R. Salaverría, (Coord.) Cibermedios: El impacto de internet en los medios de comunicación. Sevilla: Comunicación social. Ediciones y Publicaciones, pp. 39-82.

Mauri, M. y Ramon, X. (2015) "Nuevos sistemas de rendición de cuentas de la información periodística. Exploración del escenario online español". El profesional de la información, 24 (4): 380-389. https://goo.gl/pdxNwG [consulta 14/09/2017].

Mayntz, R.; Holm, K. y Hübner, P. (1993) Introducción a los métodos de la sociología empírica. Madrid: Alianza Editorial.

Morate, I. (2010) Medios y cibermedios desde la perspectiva autonómica. Situación actual y tendencias en Castilla-La Mancha. Ciudad Real: Diputación provincial de Ciudad Real.

Ortega, R. y Sánchez, I. (2007) De las gacetas al .com: la prensa en la provincia de Ciudad Real (1812-2007). Ciudad Real: Centro de Estudios de Castilla-La Mancha.

Picard, R. G. (2004) “Commercialism and Newspaper Quality". En Newspaper Research Journal, 21 (1): 54-66.

Rodríguez-Martínez R.; Codina, L. y Pedraza R. (2012) "Indicadores para la evaluación de la calidad en cibermedios: análisis de la interacción y de la adopción de la Web 2.0". Revista Española de Documentación Científica, 35(1): 61-93, DOI: https://doi.org/10.3989/redc.2012.1.858

Romero Rodríguez, L.M.; De Casas Moreno, P. y Torres Toukoumidis, A. (2016) "Dimensiones e indicadores de la calidad informativa en los medios digitales". Comunicar, 49: 91-100. https://goo.gl/HkskP4 [consulta 22/8/2018].

Rosique, G. y Barranquero, A. (2016) "Radiografía de los observatorios de medios en España. Una herramienta ciudadana para la reforma mediática". Revista Latina de Comunicación Social, 71: 443469, DOI: https://doi.org/10.4185/RLCS-2016-1104

Sánchez, I. (1991) La prensa en Castilla-La Mancha. Características y estructura (1811-1939). Ciudad Real: Servicio de Publicaciones de la Universidad de Castilla-La Mancha.

Schulz, W. (2000) "Preconditions of Journalistic Quality in an Open Society". International Conference News Media and Politics: Independent Journalism, Budapest. 


\section{Breve currículo:}

\section{Belén Galletero Campos}

Licenciada en Periodismo por la Universidad de Navarra, máster en Comunicación política y empresarial por la Universidad Camilo José Cela y doctora por la Universidad de Castilla-La Mancha (UCLM). Profesora en la Facultad de Periodismo de la UCLM e imparte docencia en el área de Redacción periodística. Ha trabajado en medios de comunicación y forma parte del grupo de investigación MediaCom, dedicado a estudiar la evolución reciente del sistema mediático en la Comunidad Autónoma de Castilla-La Mancha.

\section{Vanesa Saiz Echezarreta}

Profesora y Vicedecana de la Facultad de Periodismo de la Universidad de Castilla-La Mancha. Docente en el área de Teoría de la Comunicación y de la Información, Técnicas de investigación, Semiótica y Análisis del discurso. Especialista en Feminismo y estudios de género, representaciones y movimientos sociales. Docente en el Máster de Análisis sociocultural de la comunicación y del conocimiento (UCM) y miembro del Grupo de Investigación Consolidado Semiótica, comunicación y cultura. Investigadora del proyecto $\mathrm{I}+\mathrm{D}$ en "Problemas públicos y controversias: diversidad y participación en la esfera mediática" (CSO2017-82109-R). Es miembro del Observatorio MediaCom de la UCLM. 